\title{
Tapering with Pharmaceutical GHB or Benzodiazepines for Detoxification in GHB-Dependent Patients: A Matched-Subject Observational Study of Treatment-as-Usual in Belgium and The Netherlands
}

\author{
Harmen Beurmanjer ${ }^{1,2}$. J. J. Luykx ${ }^{3}$ - B. De Wilde ${ }^{3,4} \cdot$ K. van Rompaey ${ }^{3}$. V. J. A. Buwalda ${ }^{1}$. C. A. J. De Jong ${ }^{2}$. \\ B. A. G. Dijkstra ${ }^{1,2} \cdot$ A. F. A. Schellekens ${ }^{2,5}$
}

Published online: 21 April 2020

(c) The Author(s) 2020

\begin{abstract}
Background The gamma-hydroxybutyric acid (GHB) withdrawal syndrome often has a fulminant course, with a rapid onset and swift progression of severe complications. In clinical practice, two pharmacological regimens are commonly used to counteract withdrawal symptoms during GHB detoxification: tapering with benzodiazepines (BZDs) or tapering with pharmaceutical GHB. In Belgium, standard treatment is tapering with BZDs, while in the Netherlands, pharmaceutical GHB is the preferred treatment method. Though BZDs are cheaper and readily available, case studies suggest GHB tapering results in less severe withdrawal and fewer complications.

Objectives This study aimed to compare two treatments-as-usual in tapering methods on withdrawal, craving and adverse events during detoxification in GHB-dependent patients.

Methods In this multicentre non-randomised indirect comparison of two treatments-as-usual, patients with GHB dependence received BZD tapering (Belgian sample: $n=42$ ) or GHB tapering (Dutch sample: $n=42$, matched historical sample). Withdrawal was assessed using the Subjective and Objective Withdrawal Scales, craving was assessed with a Visual Analogue Scale and adverse events were systematically recorded. Differences in withdrawal and craving were analysed using a linear mixed-model analysis, with 'days in admission' and 'detoxification method' as fixed factors. Differences in adverse events were analysed using a Chi-square analysis.

Results Withdrawal decreased over time in both groups. Withdrawal severity was higher in patients receiving BZD tapering (subjective mean $=36.50$, standard deviation $=21.08$; objective mean $=8.05$, standard deviation $=4.68$ ) than in patients receiving pharmaceutical GHB tapering (subjective mean $=15.90$; standard deviation $=13.83$; objective mean $=3.72$; standard deviation =2.56). No differences in craving were found. Adverse events were more common in the BZD than the GHB group, especially delirium (20 vs $2.5 \%$, respectively).

Conclusions These results support earlier work that BZD tapering might not always sufficiently dampen withdrawal in GHBdependent patients. However, it needs to be taken into account that both treatments were assessed in separate countries. Based on the current findings, tapering with pharmaceutical GHB could be considered for patients with GHB dependence during detoxification, as it has potentially less severe withdrawal and fewer complications than BZD tapering.
\end{abstract}

Harmen Beurmanjer

Harmen.beurmanjer@novadic-kentron.nl

Extended author information available on the last page of the article 


\section{Key Points}

Tapering with pharmaceutical gamma-hydroxybutyric acid (GHB) led to a milder withdrawal syndrome than tapering with benzodiazepines in patients with GHB use disorder during detoxification.

Patients with GHB use disorder had a one in five chance of developing delirium during detoxification when tapering with benzodiazepines, compared with 1 in 40 when tapering with pharmaceutical GHB.

There were no differences in GHB craving levels during detoxification between patients with GHB use disorder receiving pharmaceutical GHB tapering or benzodiazepine tapering.

\section{Introduction}

Gamma-hydroxybutyric acid (GHB) is a short-chain fatty acid biosynthetically derived from the inhibitory neurotransmitter gamma-aminobutyric acid (GABA) [1], it binds to GHB and GABA-B receptors [2]. GHB is mainly used in Australia, the USA and Europe [3-5] for its euphoric and sedating effects [6-8]. GHB has a very narrow bandwidth between the plasma concentrations for desired clinical effects and overdose, often resulting in temporary coma $[9,10]$. A GHB overdose can however be fatal, especially when combined with other substances [9]. Regular GHB use can lead to GHB use disorder (GUD) [6]. While the prevalence of GHB use is still limited in Europe, between 0.1 and $1.5 \%$ of the adult population, it has been rising in the past decade [6]. Little is known about the number of people with GUD, but it is estimated that up to $21 \%$ of GHB users develops GUD [10]. GHB use disorder is characterised by frequent GHB administration (every $1-3 \mathrm{~h}$ ) to prevent withdrawal [6]. The GHB withdrawal syndrome often has a fulminant course, with a rapid onset and swift progression of severe withdrawal symptoms [6, 7]. Withdrawal symptoms include: tremor, nausea, vomiting, tachycardia, insomnia, diaphoresis, anxiety and nystagmus. Adverse events during withdrawal include hypertensive crisis, severe agitation, delirium and epileptic seizures [6].

The severity and complexity of GHB withdrawal pose clinical challenges during detoxification. In clinical practice, two pharmacological treatment regimens are commonly used to counteract withdrawal symptoms during GHB detoxification: tapering with benzodiazepines (BZDs) and tapering with pharmaceutical GHB. Benzodiazepines have an allosteric effect on GABA-A receptors, resulting in an increased sensitivity for GABA [11]. The benefits of BZDs compared with pharmaceutical GHB are the wide availability in medical settings, low costs and that tapering with BZDs allows patients to directly quit using GHB. However, several case studies describe BZD resistance [12], where despite extremely high doses of BZDs, in one case up to $700 \mathrm{mg}$ of diazepam per day, delirium still develops [13, 14]. Others describe the necessity of additional sedating medication, such as phenobarbital [15] and propofol [16], to treat delirium.

Pharmaceutical GHB has the same pharmacological properties as 'street GHB'. GHB-assisted tapering requires up to 12 doses (every $2 \mathrm{~h}$ ) a day [17]. GHB tapering has been shown to be associated with a high success rate of $85 \%$ and limited adverse events in several large non-randomised trials $(n=450)$. Reported adverse events during detoxification were mainly hypertension $(7 \%)$ and delirium $(2 \%)[17,18]$. It is suggested that tapering with pharmaceutical GHB might be preferable over BZD treatment, owing to its pharmacological similarity with street GHB. Benzodiazepines mainly act at GABA-A receptors, whereas GHB mainly acts at GHB and GABA-B receptors. Benzodiazepines might therefore be less effective in supressing GHB withdrawal because they target different receptors to GHB. A disadvantage of GHB tapering is its shorter half-life, requiring GHB administration throughout the night, which interferes with sleep. Continued GHB use could also be seen as reinforcing compulsive substance use, potentially maintaining symptoms, such as craving [19].

While both methods are currently in use, studies comparing both methods are not available. This study aimed to indirectly compare these two tapering methods for the detoxification of GHB in patients with GUD. In Belgium, physicians cannot prescribe pharmaceutical GHB for GHB withdrawal and BZD tapering is the standard of care. In the Netherlands, tapering with pharmaceutical GHB detoxification is the preferred option, based on the existing literature and national guidelines. Therefore, the current study made a matched comparison between the two treatmentsas-usual in each country. Based on the current literature and the pharmacological profile of GHB, it is expected that the pharmaceutical GHB tapering has (1) a less severe withdrawal syndrome, (2) fewer adverse events and (3) higher craving levels during the detoxification process in patients with GUD, compared with tapering with BZDs. 


\section{Methods}

\subsection{Study Design}

The study was a multicentre non-randomised indirect comparison of two treatments-as-usual, comparing the effectiveness between BZD tapering in Belgium and GHB tapering in the Netherlands in patients with GUD. Ethical approval for the Belgian part was obtained from the Institutional Review Board Ziekenhuis Netwerk Antwerpen/OCMW Antwerpen (E.C. Approval No. 4664). For the Dutch part, the Medical Ethical Research Committee Twente and Central Committee on Research Involving Human Subjects (CCMO) approved the pharmaceutical GHB protocol and considered that the study did not fall under the scope of the Medical Research Involving Human Subjects Act (WMO). The data from this study were published in 2017 [17] and a sample from this dataset is used in the current study. Off-label use of pharmaceutical GHB for GHB detoxification was approved by the Dutch Health Care Inspection and is now considered the standard detoxification treatment for patients with GUD in the Netherlands. The authors assert that all procedures contributing to this work comply with the ethical standards of the relevant national and institutional committees on human experimentation and with the Helsinki Declaration of 1975, as revised in 2008. All procedures involving human patients were approved by the above-mentioned boards and committees.

\subsection{Participants}

Participants were patients with GUD who received an indication for inpatient detoxification. Patients were included if they had (1) a diagnosis of GHB dependence according to the Diagnostic and Statistical Manual of Mental Disorders, 4th Edition, Text Revised, and (2) were aged $>18$ years at the time of admission to the hospital. Patients were excluded if they were not able to complete the study questionnaires, e.g. because of insufficient knowledge of the Dutch language or in the case of a severe acute psychiatric co morbidity that required immediate medical attention that interfered with study participation (e.g. delirium, mania, psychosis or suicidal tendencies). Participants for BZD treatment were those admitted to the psychiatric ward of the Sint-Erasmus Hospital [part of Ziekenhuis Netwerk Antwerpen in Belgium] ( $n=42)$ between October 2015 and May 2018, where BZD tapering is treatment-as-usual. Participants for pharmaceutical GHB tapering were selected from a historical sample $(n=229)$ previously recruited, between March 2011 and December 2012, from six addiction treatment centres in the Netherlands (IrisZorg, Novadic-Kentron, Tactus, Victas, Mondriaan GGZ and Verslavingszorg Noord Nederland)
[17]. Based on this sample, a matched group $(n=42)$ was selected. For matching, a three-step approach was used. First, based on the Belgium sample, the range for age, months of daily GHB use and the daily dose of GHB before admission were determined. Second, all patients within the historical comparison group of pharmaceutical GHB tapering who scored outside these ranges on one or more variables were excluded. Next, we drew a random sample of 42 patients from this comparison sample. Written informed consent was obtained from all patients prior to data collection in both samples.

\subsection{Measurements}

\subsubsection{Sociodemographics and Substance Use}

Demographics and other clinical data were obtained from chart reviews (admission data, discharge data and the discharge summary). Substance use and classification of substance dependence were assessed using the Measurement in the Addictions for Triage and Evaluation section one [20]. The section defines participants' current substance use (lasting 30 days) and lifetime substance use. For GHB use, the GHB questionnaire was used [17]. This GHB questionnaire assesses the pattern of GHB use, including the total years of use, daily dose in millilitres, millilitre per dose and time interval between doses.

\subsubsection{Withdrawal Symptoms}

Withdrawal severity was assessed using the Subjective and Objective Withdrawal Scale (SWS/OWS) [21]. The SWS, a 5-point Likert scale (0-4) with a maximum score of 132, is completed by patients and consists of 33 items related to withdrawal. It includes both mental and physical withdrawal items such as "I feel anxious," "I see things that aren't there," "I'm trembling" and "I'm tired". The OWS is completed by the nursing staff and is based on clinical observations. The scale consists of 33 items scored dichotomously (yes or no).

\subsubsection{Craving}

The Visual Analogue Scale was used to asses craving on a $0-10$ scale. Patients ticked the number that applied to their current experienced level of craving. The Visual Analogue Scale is widely used in health research, and is commonly used in studies to assess the severity of craving in patients with substance use disorders $[17,22]$.

\subsubsection{Adverse Events}

After detoxification, patients and staff were asked to completed a discharge questionnaire [17] to identify adverse 
events. In addition, discharge summaries were reviewed for any adverse events or other issues that emerged during detoxification. Adverse events were interpreted as untoward medical occurrences in a participant, such as delirium, other events resulting in severe discomfort for the patients, lifethreatening situations or admittance to an intensive care unit.

\subsection{Detoxification Treatment}

\subsubsection{Detoxification with Benzodiazepines}

Diazepam (or lorazepam when serious liver disease was present) was titrated based on vital parameters. Vitals were measured once per $30 \mathrm{~min}$. When blood pressure rose above $140 / 90 \mathrm{mmHg}$ and/or heart rate rose above 100 beats per minute, diazepam $10 \mathrm{mg}$ (or lorazepam $2.5 \mathrm{mg}$ ) was administered. In the event of a more than $20 \mathrm{mmHg}$ rise in pressure (systolic or diastolic blood) and/or a 20 beats per minute increase in heart rate, the diazepam dose was increased with $20 \mathrm{mg}$ (or lorazepam $5 \mathrm{mg}$ ). The dose of diazepam (or lorazepam) was adjusted every $30 \mathrm{~min}$, if needed, until blood pressure and heart rate dropped below 140/90 $\mathrm{mmHg}$ and 100 beats per minute, respectively. The total detoxification and tapering schedule took on average 7 days. Vitals were measured once every $30 \mathrm{~min}$ in the first $48 \mathrm{~h}$ of BZD detoxification, and after that vitals were measured every 3-4 $\mathrm{h}$, until the end of detoxification.

\subsubsection{Detoxification with Pharmaceutical Gamma-Hydroxybutyric Acid}

Detoxification started with a titration phase, where patients were treated with pharmaceutical GHB on $70 \%$ of their street GHB dose. Next, the GHB dose was titrated up in case of withdrawal and titrated down in case of sedation, until the right pharmaceutical GHB dose was found on which patients were stable and experienced neither withdrawal nor sedation. This usually took between 1 and 2 days, after which the tapering phase started. During the tapering phase, the GHB dose was lowered by $300 \mathrm{mg}$ of GHB per given dose per day. The interval between doses was usually $2 \mathrm{~h}$, or up to $3 \mathrm{~h}$ depending on withdrawal severity. For a more detailed description of the protocol, see [17]. The total detoxification and tapering schedule took on average 11 days.

\subsubsection{Treating Delirium}

In the case of delirium, an atypical antipsychotic (quetiapine, olanzapine) or haloperidol was prescribed in the Netherlands [17]. In Belgium, clotiapine was prescribed in addition to the aforementioned medications.

\subsection{Procedure}

After signing the informed consent, the Measurement in the Addictions for Triage and Evaluation and GHB questionnaires were completed. During detoxification, withdrawal symptoms and craving were monitored three times a day. After the detoxification process, and before discharge from the hospital, the discharge questionnaire was completed.

\subsection{Analysis}

Group differences in baseline characteristics were compared using a one-way analysis of variance for continuous variables and a Chi-square analysis for non-continuous variables. A linear mixed-model analysis was used with withdrawal (SWS, OWS) and craving (Visual Analogue Scale craving) scores as dependent variables and 'days in admission' (within-subjects variable) and 'detoxification method of use' (between-subjects variable) as fixed factors. For all three questionnaires, average scores per patient per day were calculated, to avoid daytime variation. Because BZD tapering lasted 7 days on average, we only analysed differences in withdrawal severity and craving between the two conditions over the first 7 days of detoxification. Adverse events and dropout rates were compared between groups by a Chi square test. $P$-values $<0.05$ (two sided) were considered statistically significant. Data were analysed with IBM SPSS Statistics for Windows, Version 24.0. Armonk, NY, USA.

\section{Results}

\subsection{Patient Characteristics}

A total of 84 patients $(\approx 70 \%$ male) were analysed in this study: 42 received BZDs and 42 received pharmaceutical GHB during their detoxification. The groups did not

Table 1 Patient characteristics of benzodiazepines (BZDs) and pharmaceutical gamma-hydroxybutyric acid (GHB) detoxification groups

\begin{tabular}{lcll}
\hline & BZD $(n=42)$ & $\begin{array}{l}\text { Pharmaceuti- } \\
\text { cal GHB } \\
(n=42)\end{array}$ & $p$ \\
\hline Male: $n(\%)$ & $29(69.0 \%)$ & $31(73.8 \%)$ & 0.801 \\
Age: years (SD) & $30.5(5.5)$ & $28.7(5.6)$ & 0.130 \\
Months of daily GHB use & $44.8(46.4)$ & $51.1(33.4)$ & 0.493 \\
(SD) & $76.7(61.9)$ & $75.7(52.4)$ & 0.953 \\
Daily ml GHB (SD) & $92.3 \%$ & $94.2 \%$ & 0.702 \\
Co-morbid substance use (in past thirty days) & & 0.090 \\
Nicotine & $76.0 \%$ & $59.1 \%$ & 0.637 \\
Alcohol & $55.6 \%$ & $60.4 \%$ & \\
Stimulants & & &
\end{tabular}

$S D$ standard deviation 
Table 2 Prescribed benzodiazepine (BZDs) and pharmaceutical gamma-hydroxybutyric acid (GHB) dose during detoxification in both groups

\begin{tabular}{llc}
\hline Day & $\begin{array}{l}\text { Average GHB dose in grams } \\
(\text { SD) }\end{array}$ & $\begin{array}{l}\text { Average diazepam } \\
\text { dose in mg (SD) }\end{array}$ \\
\hline 1 & $28.24(10.51)$ & $60.32(38.61)$ \\
2 & $25.01(10.07)$ & $100.34(64.15)$ \\
3 & $22.13(9.83)$ & $74.16(45.77)$ \\
4 & $19.60(9.24)$ & $63.55(27.95)$ \\
5 & $18.08(8.68)$ & $51.86(25.44)$ \\
6 & $16.12(8.38)$ & $44.74(38.10)$ \\
7 & $14.02(6.47)$ & $33.74(29.76)$ \\
8 & $11.56(5.94)$ & \\
9 & $8.46(5.66)$ & \\
10 & $6.28(6.93)$ & \\
11 & $4.80(3.25)$ & \\
\hline
\end{tabular}

$S D$ standard deviation

differ on demographic and GHB use characteristics. While all patients used multiple substances in the 30 days before admission, no differences between the groups were found (see Table 1). Nicotine, stimulants and alcohol were most commonly used besides GHB by participants in both groups in the past 30 days. An overview of BZD and GHB doses prescribed during detoxification for both groups is shown in Table 2.

\subsection{Subjective Withdrawal Symptoms}

The linear mixed-model analysis showed that patients reported a decrease in withdrawal symptoms (SWS) over time during the detoxification (main effect of time: $F(6,11)=6.481, p<0.003)$. Patients in the pharmaceutical GHB group had lower SOS scores (mean $=15.90$; standard deviation $[\mathrm{SD}]=13.83$ ) than those in the BZD group (mean $=36.50 ;$ SD 21.08), indicating less severe withdrawal in the GHB group (main effect of group: $F(1,1688)=42.336$, $p<0.001$ ), see Fig. 1. No interaction effect between group and time was found. As BZD tapering lasted 7 days on average, the comparison of subjective withdrawal is shown only over the first 7 days of detoxification. The results did not differ when the full 11 days of pharmaceutical GHB tapering were included in the analysis.

\subsection{Objective Withdrawal Symptoms}

The linear mixed-model showed that withdrawal symptoms (OWS) scored by the staff decreased over time during detoxification in both groups (main effect of time: $F(6,50)=6.7, p<0.001)$. Patients in the pharmaceutical GHB group showed a lower severity of withdrawal symptoms (mean $=3.72$; SD 2.56) compared with the BZD group (mean $=8.05 ;$ SD 4.68), indicating less severe withdrawal in the GHB group (main effect of group: $F(1,102)=39.2$, $p<0.001)$, see Fig. 2. There was an interaction effect between time and group $(F(6,50)=3.0, p<0.05)$, indicating that the observed withdrawal symptoms pattern differs between the two treatments over time. This interaction effect is mainly driven by an increase in withdrawal severity on day 3 of admission in the BZD group, see Fig. 2. As BZD tapering lasted 7 days on average, the comparison of objective withdrawal is shown only over the first 7 days of detoxification. The results did not differ when the full 11 days of pharmaceutical GHB tapering were included in the analysis.

\subsection{Craving}

The linear mixed-model showed that craving diminished over time during detoxification in both groups (main effect of time: $F(1,6)=6.88, p<0.001$ ), see Fig. 3. No differences in craving scores were found between BZD and GHB tapering. As BZD tapering lasted 7 days on average, the comparison of craving is shown only over the first 7 days
Fig. 1 Experienced subjective withdrawal (SWS) during detoxification in patients receiving benzodiazepine (BZD) tapering or pharmaceutical gammahydroxybutyric acid (GHB) tapering

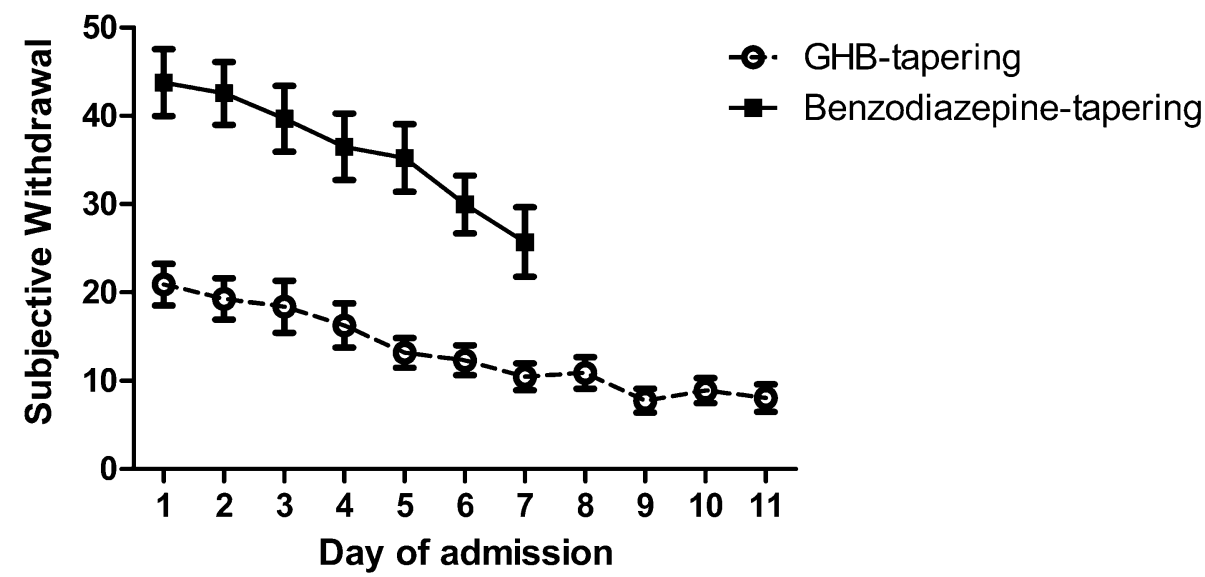


Fig. 2 Observed objective withdrawal (Objective Withdrawal Scale [OWS]) during detoxification in patients receiving benzodiazepine (BZD) tapering or pharmaceutical gammahydroxybutyric acid (GHB) tapering
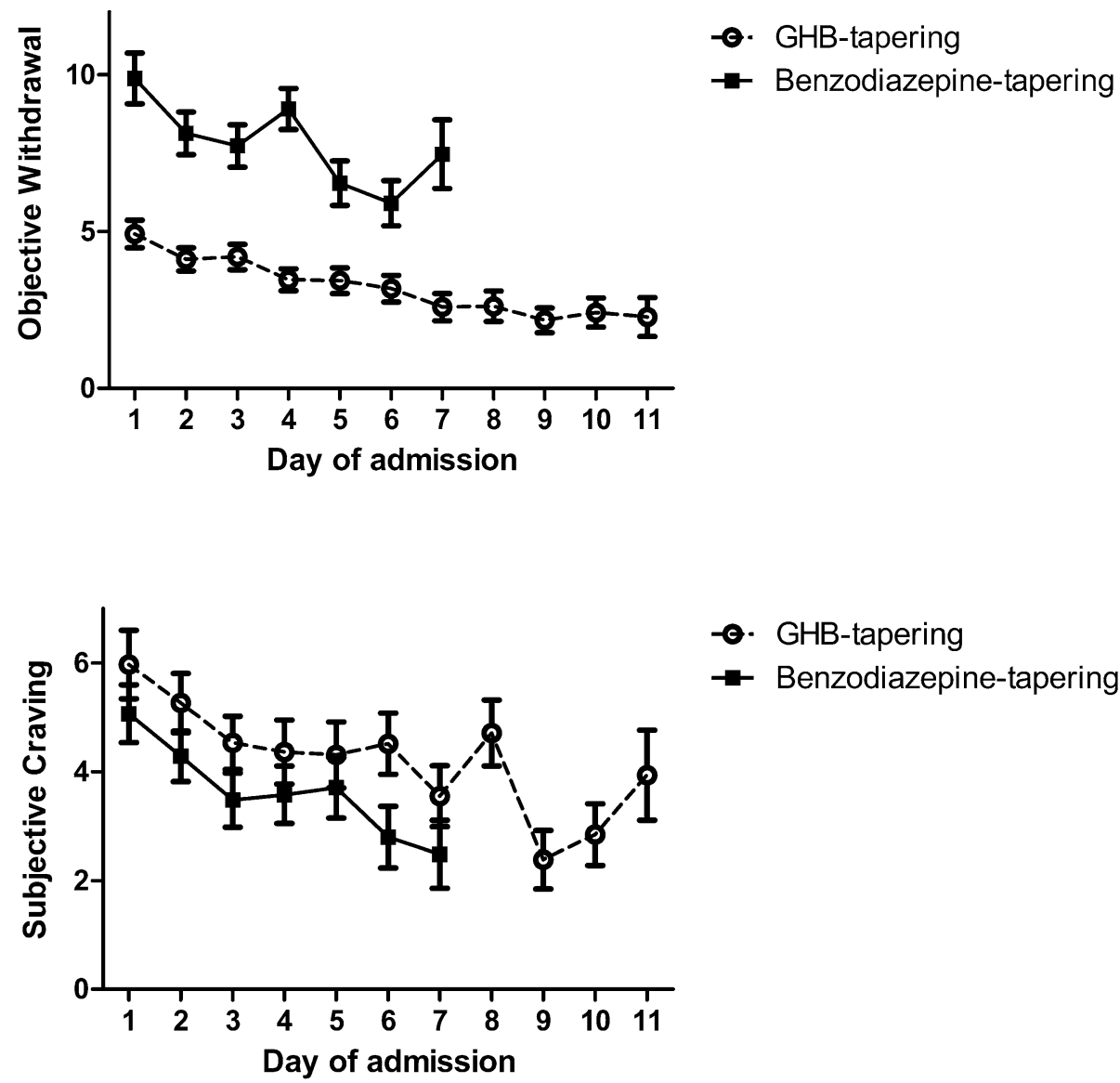

Fig. 3 Experienced craving (Visual Analogue Scale [VAS]) during detoxification in patients receiving benzodiazepine (BZD) tapering or pharmaceutical gamma-hydroxybutyric acid (GHB) tapering

\section{Discussion}

of detoxification. The results did not differ when the full 11 days of pharmaceutical GHB tapering were included in the analysis.

\subsection{Adverse Events}

Adverse events were more common in the BZD group, with $29 \%(n=12)$ of BZD-treated patients developing an adverse event during detoxification, compared with $5 \%(n=2)$ of the patients in the pharmaceutical GHB group $\left(\chi^{2}(1)=8.5714\right.$, $p=0.003)$. The majority of adverse events was related to delirium. Delirium was more common in the BZD group $(21 \%, n=9)$, compared with the pharmaceutical GHB group $(2 \%, n=1)\left[\chi^{2}(1)=7.2649, p=0.007\right]$. Two patients receiving BZD tapering were transferred to the intensive care unit of the hospital, after developing delirium. Both patients developed severe agitation during their delirium. Other reported adverse events were latent suicidal thoughts, severe nightmares and memory problems in the BZD group.
This study compared BZD and GHB tapering in patients with GUD. In line with the hypotheses, GHB tapering was associated with a milder withdrawal syndrome and fewer adverse events (including delirium) during detoxification, compared with BZD treatment. Contrary to our hypothesis, no differences in craving scores between the two groups were found. These findings suggest that tapering with pharmaceutical GHB might be more comfortable and safer than detoxification with BZDs in patients with GUD. The difference in reported adverse events, in particular delirium, is highly relevant from a clinical perspective. In the GHB group, one in 40 patients developed delirium, in comparison to one in five patients in the BZD group.

Benzodiazepines might not sufficiently counteract GHB withdrawal symptoms in all patients $[12,14,23]$. The current findings are in line with several case studies on detoxification of patients with GUD, where delirium was common in BZD tapering, despite very high BZD doses [6, 13, 14, 25]. This difference might be explained by the different working mechanism of BZDs and GHB. Where BZDs primarily affect GABA-A receptors [11], GHB binds to GABA-B and GHB receptors [2]. Withdrawal symptoms of GHB, probably 
mediated through GABA-B and GHB receptors, might thus not be sufficiently suppressed through BZDs acting through GABA-A. Furthermore, BZD use has been associated with the development of delirium in some cases [24]. This might have further contributed to the increased risk of delirium in the BZD group.

In the present study, withdrawal symptoms were more severe in patients treated with BZDs from the start of detoxification, compared with patients treated with pharmaceutical GHB. This might be related to differences in the titration procedure between the two conditions. Where initiation of BZD administration was based on increased blood pressure and/or heart rate (symptom triggered), pharmaceutical GHB administration was initiated $2 \mathrm{~h}$ after the last ingestion of street GHB, and then continued every $2 \mathrm{~h}$ in a fixed schedule. Furthermore, patients might develop withdrawal symptoms before their blood pressure and/or heart rate increase. Therefore, patients treated with pharmaceutical GHB might have experienced fewer withdrawal symptoms from the start of detoxification than patients treated with BZDs. Therefore, differences in the titration procedure between the conditions might also have contributed to the differences in withdrawal severity that we observed.

Another important difference between BZD and GHB detoxification is the duration of the detoxification. Tapering with BZDs took on average 7 days and tapering with GHB took 11 days. GHB detoxification thus seems more gradual, and might therefore be associated with fewer withdrawal symptoms and adverse events, including delirium, as compared with BZD tapering. The observed increased withdrawal severity and risk for delirium in the BZD group might thus also be related to more rapid detoxification with BZD tapering, compared with GHB tapering. As the vast majority of patients receiving BZD tapering did not experience any adverse events and had on average a shorter detoxification period, it could be argued that pharmaceutical GHB tapering could also be achieved in 7 days. However, this will likely increase withdrawal severity and possibly also the risk of adverse events such as delirium.

Some additional considerations regarding both treatment options as assessed in this study should be taken into account. First, tapering with GHB requires frequent administration of doses during the entire day and often also during the night. This is a demanding procedure for both the patient and staff. However, prevention of delirium and other adverse events of GHB withdrawal clearly outweighs the burden for patients and staff. Second, the length of admission in the GHB tapering group was shorter than reported in other publications in the Dutch GHB sample [17, 18]. The discrepancy in the length of stay between this study and past GHB studies with the Dutch sample is likely accounted by the matching process, as the Belgium sample used, on average, a lower GBH dose than commonly reported in the Netherlands
[17]. Patients using higher doses of street GHB are more likely to experience severe withdrawal and adverse events such as delirium during detoxification and BZDs are less effective in preventing delirium in this population. Given that our study participants use lower-than-average doses of street GHB prior to detoxification, the current findings may be an underestimation of the beneficial effects of pharmaceutical GHB compared to BZDs in more severe GUD populations.

Last, substitution of 'street' GHB with pharmaceutical GHB means that patients have to continue to use a substance that they are trying to quit. It can be speculated that this continued use could reinforce GHB use and sustain the compulsive pattern of use [19]. However, no differences in craving levels were found between the two groups, indicating that patients in the GHB group did not experience a stronger need to use than patients in the BZD group. It would be interesting to study if the type of detoxification influences relapse rates after detoxification in future longitudinal studies.

While future studies would ideally use randomised controlled designs to replicate our findings, this might not be feasible in this patient population for several reasons. First, patients with GUD often receive treatment in acute situations requiring immediate care because of a fast-developing severe withdrawal syndrome. This complicates informed consent and randomisation procedures. Second, the results from the current study in combination with the existing literature point towards potentially high risks of complications during BZD tapering in patients with GUD. This further complicates randomisation to BZD vs GHB tapering from an ethical point of view, as BZD tapering might be inferior to pharmaceutical GHB tapering. The current comparative study was mainly possible because of juridical restrictions of pharmaceutical GHB use in Belgium, offering the possibility of an observational non-randomised trial.

Future research on GHB detoxification should also focus on optimisation of the duration of detoxification and study whether some patients could profit more from one method or the other. For instance, it is likely that in patients with relatively low levels of GHB use, BZD tapering might be sufficient to counteract GHB withdrawal, whereas in patients using high levels of GHB, pharmaceutical GHB might be the preferred option. Cost effectiveness should also be taken into account, as pharmaceutical GHB is more expensive than BZDs.

Future studies should also focus on the GABA-B agonist baclofen as an alternative for BZD and/or GHB tapering in GUD [26, 27]. As baclofen has a longer half-life than GHB and targets GABA-B receptors, it might effectively suppress GHB withdrawal. Moreover, baclofen tapering might allow patients to quit using GHB, preventing withdrawal, with only three to four daily dosages [26, 28]. Furthermore, baclofen 
has been suggested to be effective in reducing relapse after detoxification [26, 28]. Given its similarities to GHB, baclofen might also be a candidate for GHB substitution therapy.

The current findings should be viewed in the light of some limitations of this study. Given the explorative nonrandomised design of the study and the fact that each treatment was assessed in a different country and a different institution, there is a risk for selection bias and procedural confounding, respectively. However, the two groups were matched and did not differ on key variables, such as the level of GHB use, duration of GHB use, co-morbid substance use, age and sex. Both populations were Dutch speaking, and the Netherlands and (Flemish) Belgium are culturally bound together. This minimises the risk of an effect of language and cultural differences between groups. It is also important to note that both groups received a similar treatment by experienced medical staff. No additional (psychotherapy) treatment was offered at both institutes during detoxification, ruling out the influence of one treatment being more extensive than the other. Yet, any confounding effect of selection bias or treatment institute cannot be fully ruled out. Another possible limitation is that delirium assessments were based on clinical observations, as reported in the discharge summaries written by the treating psychiatrist and in the treatment outcome forms. The use of a structured scale, such as the Delirium Observation Screening Scale, might have been more reliable [29].

\section{Conclusions}

In patients with GUD, detoxification with pharmaceutical GHB showed less severe withdrawal symptoms and fewer adverse events, specifically delirium, than detoxification with BZDs. This supports earlier work that BZDs might not always sufficiently dampen withdrawal in GUD [6, 13, 14]. Based on the current findings, tapering with pharmaceutical GHB could be considered for patients with GHB dependence during detoxification, as it has potentially less severe withdrawal and fewer complications than BZD tapering.

Acknowledgements The authors thank the participating addiction treatment centres, as well as all of the individuals who assisted in this study. In particular, we thank the study nurses and patients for their effort and willingness. The authors thank Dr. Bernd Figner from the Radboud University Nijmegen for help with the data analysis.

Author Contributions Jurjen Luykx, Bieke de Wilde and Koen van Rompaey managed the data collection in Belgium. Harmen Beurmanjer and Boukje Dijkstra managed the data collection in the Netherlands. Arnt Schellekens and Harmen Beurmanjer conducted most of the analysis and wrote the first draft of the manuscript. Victor Buwalda and Cor de Jong were involved in the data interpretation and supervision of the project. All authors critically revised the manuscript and gave final approval of the version to be submitted.

\section{Compliance with Ethical Standards}

Funding The Netherlands Ministry of Health, Welfare and Sports (VWS) funded the collection of the Dutch data [316997] within the framework of the national programme of the Dutch Association of Mental Health and Addiction Care: 'Scoring results'. They had no role in the execution of the project and interpretation of the results. The open access fee was paid by the Radboud University Nijmegen.

Conflict of interest Harmen Beurmanjer, Jurjen J. Luykx, Bieke De Wilde, Koen van Rompaey, Victor J.A. Buwalda, Cor A.J. De Jong, Boukje A.G. Dijkstra and Arnt F.A. Schellekens have no conflicts of interest that are directly relevant to the content of this study.

Open Access This article is licensed under a Creative Commons Attribution-NonCommercial 4.0 International License, which permits any non-commercial use, sharing, adaptation, distribution and reproduction in any medium or format, as long as you give appropriate credit to the original author(s) and the source, provide a link to the Creative Commons licence, and indicate if changes were made. The images or other third party material in this article are included in the article's Creative Commons licence, unless indicated otherwise in a credit line to the material. If material is not included in the article's Creative Commons licence and your intended use is not permitted by statutory regulation or exceeds the permitted use, you will need to obtain permission directly from the copyright holder.To view a copy of this licence, visit http://creativecommons.org/licenses/by-nc/4.0/.

\section{References}

1. Tarabar AF, Nelson LS. The gamma-hydroxybutyrate withdrawal syndrome. Toxicol Rev. 2004;23(1):45-9.

2. Laborit H. Sodium 4-hydroxybutyrate. Int J Neuropharmacol. 1964;3:433-51.

3. Degenhardt L, Darke S, Dillon P. The prevalence and correlates of gamma-hydroxybutyrate (GHB) overdose among Australian users. Addiction. 2003;98(2):199-204.

4. Dines AM, Wood DM, Yates C, Heyerdahl F, Hovda KE, Giraudon I, et al. Acute recreational drug and new psychoactive substance toxicity in Europe: 12 months data collection from the European Drug Emergencies Network (Euro-DEN). Clin Toxicol. 2015;53(9):893-900.

5. Zvosec DL, Smith SW, Hall BJ. Three deaths associated with use of Xyrem. Sleep Med. 2009;10(4):490-3.

6. Kamal RM, van Noorden MS, Wannet W, Beurmanjer H, Dijkstra BAG, Schellekens A. Pharmacological treatment in gammahydroxybutyrate (GHB) and gamma-butyrolactone (GBL) dependence: detoxification and relapse prevention. CNS Drugs. 2017;31(1):51-64.

7. Busardo F, Jones A. GHB pharmacology and toxicology: acute intoxication, concentrations in blood and urine in forensic cases and treatment of the withdrawal syndrome. Curr Neuropharmacol. 2015;13(1):47-70.

8. Beurmanjer H, Asperslag EM, Oliemeulen L, Goudriaan AE, De Jong CAJ, Schellekens ASA, et al. A qualitative approach in understanding illness perception and treatment needs in patients with gamma hydroxybutyrate use disorder. Eur Addict Res. 2019. https://doi.org/10.1159/000500807. 
9. Corkery JM, Loi B, Claridge H, Goodair C, Corazza O, Elliott $\mathrm{S}$, et al. Gamma hydroxybutyrate (GHB), gamma butyrolactone (GBL) and 1,4-butanediol (1,4-BD; BDO): a literature review with a focus on UK fatalities related to non-medical use. Neurosci Biobehav Rev. 2015;53:52-78. https://doi.org/10.1016/j.neubi orev.2015.03.012.

10. Miotto K, Darakjian J, Basch J, Murray S, Zogg J, Rawson R. Gamma-hydroxybutyric acid: patterns of use, effects and withdrawal. Am J Addict. 2001;10(3):232-41.

11. Lorenz-Guertin JM, Bambino MJ, Das S, Weintraub ST, Jacob TC. Diazepam accelerates GABAAR synaptic exchange and alters intracellular trafficking. Front Cell Neurosci. 2019;13:163. https ://doi.org/10.3389/fncel.2019.00163.

12. van Noorden MS, Kamal RM, Dijkstra BAG, Mauritz R, de Jong CAJ. A case series of pharmaceutical gamma-hydroxybutyrate in 3 patients with severe benzodiazepine-resistant gammahydroxybutyrate withdrawal in the hospital. Psychosomatics. 2015;56(4):404-9.

13. Craig K, Gomez HF, McManus JL, Bania TC. Severe gammahydroxybutyrate withdrawal: a case report and literature review. J Emerg Med. 2000;18(1):65-70.

14. Neu P. Course and complications of GHB detoxification treatment: a 1-year case series [in German]. Nervenarzt. 2019;90(5):509-15. https://doi.org/10.1007/s00115-018-0636-8.

15. Sivilotti MLA, Burns MJ, Aaron CK, Greenberg MJ. Pentobarbital for severe gamma-butyrolactone withdrawal. Ann Emerg Med. 2001;38(6):660-5.

16. Dyer JE, Roth B, Hyma BA. Gamma-hydroxybutyrate withdrawal syndrome. Ann Emerg Med. 2001;37(2):147-53.

17. Dijkstra BAG, Kamal R, van Noorden MS, de Haan H, Loonen AJM, De Jong CAJ. Detoxification with titration and tapering in gamma-hydroxybutyrate (GHB) dependent patients: the Dutch GHB monitor project. Drug Alcohol Depend. 2017;170:164-73.

18. Beurmanjer H, Verbrugge CAG, Schrijen SSA, DeJong CAJ DB. Treatment of patients with GHB dependence: end report of the GHB Monitor 2.0 [in Dutch]. Nijmegen: NISPA; 2016.

19. Kwako LE, Koob GF. Neuroclinical framework for the role of stress in addiction. Chronic Stress (Thousand Oaks). 2017 Feb; 1: https://doi.org/10.1177/2470547017698140. https://doi. org/10.1177/2470547017698140.
20. Schippers GM, Broekman TG, Buchholz A, Koeter MWJ, Van Den Brink W. Measurements in the Addictions for Triage and Evaluation (MATE): an instrument based on the World Health Organization family of international classifications. Addiction. 2010;105(5):862-71.

21. Handelsman L, Cochrane KJ, Aronson MJ, Ness R, Rubinstein $\mathrm{KJ}$, Kanof PD. Two new rating scales for opiate withdrawal. Am J Drug Alcohol Abuse. 1987;13(3):293-308.

22. Schmitz JM, Green CE, Hasan KM, Vincent J, Suchting R, Weaver MF, et al. PPAR-gamma agonist pioglitazone modifies craving intensity and brain white matter integrity in patients with primary cocaine use disorder: a double-blind randomized controlled pilot trial. Addiction. 2017;112(10):1861-8.

23. Strand NAW, Petersen TS, Nielsen LM, Boegevig S. Treatment of gamma-hydroxybutyrate withdrawal [in Danish]. Ugeskr Laeger. 2017;179(50).

24. Zaal IJ, Devlin JW, Hazelbag M, Klein Klouwenberg PMC, van der Kooi AW, Ong DSY, et al. Benzodiazepine-associated delirium in critically ill adults. Intensive Care Med. 2015;41(12):2130 7. https://doi.org/10.1007/s00134-015-4063-z.

25. Schneir AB, Ly BT, Clark RF. A case of withdrawal from the GHB precursors gamma-butyrolactone and 1,4-butanediol. J Emerg Med. 2001;21(1):31-3.

26. Beurmanjer H, Kamal RM, de Jong CAJ, Dijkstra BAG, Schellekens AFA. Baclofen to prevent relapse in gamma-hydroxybutyrate (GHB)-dependent patients: a multicentre, open-label, non-randomized, controlled trial. CNS Drugs. 2018;32(5):437-42.

27. Lingford-Hughes A, Patel Y, Bowden-Jones O, Crawford MJ, Dargan PI, Gordon F, et al. Improving GHB withdrawal with baclofen: study protocol for a feasibility study for a randomised controlled trial. Trials. 2016;17(1):472.

28. Kamal RM, Schellekens A, De Jong CAJ, Dijkstra BAG. Baclofen as relapse prevention in the treatment of gamma-hydroxybutyrate (GHB) dependence: an open label study. BMC Psychiatry. 2015;15(1):1-8.

29. Schuurmans MJ, Shortridge-Baggett LM, Duursma SA. The Delirium Observation Screening Scale: a screening instrument for delirium. Res Theory Nurs Pract. 2003;17(1):31-50.

\section{Affiliations}

\section{Harmen Beurmanjer ${ }^{1,2} \cdot$ J. J. Luykx ${ }^{3}$ B. De Wilde ${ }^{3,4} \cdot K^{\prime}$ van Rompaey ${ }^{3}$ V. J. A. Buwalda ${ }^{1}$. C. A. J. De Jong ${ }^{2} \cdot$ B. A. G. Dijkstra ${ }^{1,2} \cdot$ A. F. A. Schellekens ${ }^{2,5}$}

1 Novadic-Kentron Addictioncare, Hogedwarsstraat 3, 5261, AE, Vught, The Netherlands

2 Nijmegen Institute for Scientist-Practitioners in Addiction (NISPA), Nijmegen, The Netherlands

3 Ziekenhuis Netwerk Antwerpen (ZNA), Borgerhout, Belgium

4 University Medical Center Utrecht, Utrecht, The Netherlands
5 Department of Psychiatry, RadboudUmc, Donders Institute for Brain, Cognition and Behaviour, Nijmegen, The Netherlands 